\title{
Cloning and Characterization of Two Vertebrate Homologs of the Drosophila eyes absent Gene
}

\author{
John E. Zimmerman, ${ }^{1}$ Quang T. Bui, ${ }^{1}$ Eiríkur Steingrímsson, ${ }^{2}$ \\ Deborah L. Nagle, ${ }^{3,5}$ Weili Fu, ${ }^{1}$ Anna Genin, ${ }^{4}$ Nancy B. Spinner, ${ }^{4}$ \\ Neal G. Copeland, ${ }^{2}$ Nancy A. Jenkins, ${ }^{2}$ Maja Bucan, ${ }^{3}$ and \\ Nancy M. Bonini ${ }^{1,6}$
}

\begin{abstract}
Departments of Biology, ${ }^{1}$ Psychiatry, ${ }^{3}$ and Pediatrics, ${ }^{4}$ University of Pennsylvania and the University of Pennsylvania Medical School, Philadelphia, Pennsylvania 19104; ${ }^{2}$ Mammalian Genetics Laboratory, ABL-Basic Research Program, National Cancer Institute (NCI)-Frederick Cancer Research and Development Center, Frederick, Maryland 21702
\end{abstract}

\begin{abstract}
The Drosophila eyes absent (eya) gene plays an essential role in the events that lead to proper development of the fly eye and embryo. Here we report the analysis of two human and two mouse homologs of the fly eya gene. Sequence comparison reveals a large domain of $\sim 270$ amino acids in the carboxyl terminus of the predicted mammalian proteins that shows $53 \%$ identity between the fly sequence and all of the vertebrate homologs. This Eya-homology domain is of novel sequence, with no previously identified motifs. RNA hybridization studies indicate that the mouse genes are expressed during embryogenesis and in select tissues of the adult. Both mouse Eya genes are expressed in the eye, suggesting that these genes may function in eye development in vertebrates as eya does in the fly. The mouse Eya2 gene maps to chromosome 2 in the region syntenic with human chromosome 20q13, and the mouse Eya3 gene maps to chromosome 4 in the region syntenic with human chromosome 1p36. Our findings support the notion that several families of genes (Pax-6/eyeless, Six-3/sine oculis, and Eya) play related and critical roles in the eye for both flies and vertebrates.
\end{abstract}

[The sequence data described in this paper have been submitted to GenBank under accession nos. U81601U81604.]

The Drosophila eye provides a striking example of how cell-cell interactions, cell autonomous pathways, and hormonal events merge into the generation of an exquisitely organized neural structure (Tomlinson 1989; Wolff and Ready 1993; Heberlein and Moses 1995). The study of genes that function in the fly eye has revealed insight into the developmental mechanisms and genetic interactions of many genes with homologs in vertebrates (Zipursky and Rubin 1994; Bonini and Choi 1995). In particular, the gene regulatory pathway for eye development displays remarkable conservation of molecular features from fly to human. One gene that functions critically in human eye development is the

\footnotetext{
5Present address: Millennium Pharmaceuticals, Inc., Cambridge, Massachusetts 02139.

${ }^{6}$ Corresponding author.

E-MAIL nbonini@sas.upenn.edu; FAX (215) 898-8780.
}

Aniridia gene that encodes a Pax-6 homeobox and paired-box gene (Ton et al. 1991; Glaser et al. 1992; Jordan et al. 1992). Loss of normal gene function in humans leads to eye developmental abnormalities, including loss of the iris in severe cases and cataracts in mild forms (Hanson and van Heyningen 1995). The fly counterpart of Pax- 6 is the eyeless gene (Quiring et al. 1994), which when mutated results in loss of the eye. Moreover, expression of eyeless in various tissues of the fly can direct cells down an eye developmental pathway, resulting in the formation of ectopic eyes (Halder et al. 1995). The mouse homolog of eyeless, Small eye (Hogan et al. 1986; Hill et al. 1991), can also direct the eye cell fate when transformed into the fly (Halder et al. 1995), indicating conservation of at least some molecular features of the eye developmental pathway from flies to vertebrates. Given the remarkable conservation of the role of Pax-6 homologs in eye development from 


\section{VERTEBRATE HOMOLOGS OF DROSOPHILA EYES ABSENT}

flies to vertebrates, the fly is providing an important guide to define new genes in vertebrates that may function in early developmental events of eye formation (Oliver et al. 1995).

The Drosophila eyes absent (eya) gene is essential for normal eye development (Bonini et al. 1993). With loss of eya function, all eye progenitor cells die by programmed cell death early in the differentiation process, resulting in an eyeless adult fly. The role of eya is to promote the survival and/or the differentiation of eye progenitor cells at an early step in their development. Although more is known of the role of eya in eye development, the eya gene is also required in select other tissues of the fly, such as during embryogenesis (Nüsslein-Volhard et al. 1984; Bonini et al. 1993). Select mutations of eya specifically affect its role in the eye, suggesting that these mutations define regulatory elements important for gene expression specifically in eye progenitor cells (Leiserson et al. 1994).

The sequence of the fly eya gene predicted a protein with no homologs or previously defined motifs indicative of function (Bonini et al. 1993). The protein is present in the nucleus of eye progenitor cells from early stages of development. As one approach to define new genes of relevance to vertebrate eye development, as well as to define critical functional domains within the Eya protein, we sought to define vertebrate homologs of the gene. During the course of these studies, Eya homologs were reported in the data banks (Banfi et al. 1996; EST Sequence Database) and by others (Xu et al. 1997). Here we confirm these observations and extend them by providing additional sequence data and detailed chromosomal map localizations of two vertebrate genes that show striking sequence conservation with the predicted fly Eya protein. These homologs are expressed in the mouse eye, suggesting that these genes, like their fly counterpart, may play a role in eye development.

\section{RESULTS}

\section{Isolation of Vertebrate Homologs of the Fly eya Gene}

Previously, we had defined a short region in the carboxyl terminus of the Drosophila eya gene that was highly conserved to a gene from the distantly related Drosophila species D. virilis (N. Bonini and S. Benzer, unpubl.). Subsequently, the Genexpress cDNA Program reported partial sequence of a human brain cDNA that revealed high homology in this same region with the predicted sequence of the Drosophila Eya protein. Based on the human sequence, we designed primers to attempt the ampli- fication of a probe for screening mouse and human cDNA libraries (see Methods for details). For these studies we used human genomic DNA and a human retinal cDNA library, and mouse genomic DNA and mouse brain cDNA. Amplification of an anticipated 306-bp product was achieved for both human retinal library and mouse brain cDNA. These amplification products, called H306 from human and M306 from mouse, were then used as probes to screen human retinal, human brain, mouse embryonic, and mouse retinal cDNA libraries to obtain cDNA clones. The M306 probe was also hybridized under high stringency conditions to Southern blots of mouse genomic DNA. By this analysis, M306 detected multiple strongly cross-hybridizing bands in genomic digests of mouse DNA (data not shown).

\section{Sequence Analysis of Human and Mouse Clones Highlights Conserved Features of Eya}

From the mouse embryonic cDNA library we isolated two different classes of eya-related clones; the longest of each class were sequenced. These clones represent two different homologs of the fly eya gene, which we refer to as Eya2 and Eya3; Eya1 has been identified by others (Xu et al. 1997). These cDNAs recognized a subset of the genomic fragments on mouse genomic Southern blots that were labeled with the M306 probe (data not shown). From the human brain library, we isolated a single class of human cDNA, EYA2, that contained an overlapping sequence with the Genexpress cDNA clone and was highly homologous to mouse Eya2 clones. From the human retinal library, we isolated a second class of human cDNA clones, EYA3, with strong identity with the mouse Eya3 clones. The DNA sequences and predicted amino acid translations of these clones are presented in Figures 1 and 2 .

The longest human EYA2 clone is not likely full length and contains a partial open reading frame (ORF) of 244 amino acids that is $96 \%$ identical at the predicted protein level with mouse Eya2 clones (Fig. 2C). EYA3 clones are $95 \%$ identical at the predicted amino acid level over the carboxy-terminal 407 amino acids with the mouse Eya3 class (Fig. 2D). These and other data (see Fig. 7, below; Discussion; Banfi et al. 1996) indicate that clones of the EYA2 class are likely to be the human homologs of the mouse Eya2 clones, whereas human EYA3 cDNAs are the homologs of mouse Eya3 clones. The aminoterminal-most sequence of the predicted protein corresponding to EYA3 differs from the corresponding region of the protein encoded by the longest mouse Eya3 clone that we have (Fig. 2D). These and 
A

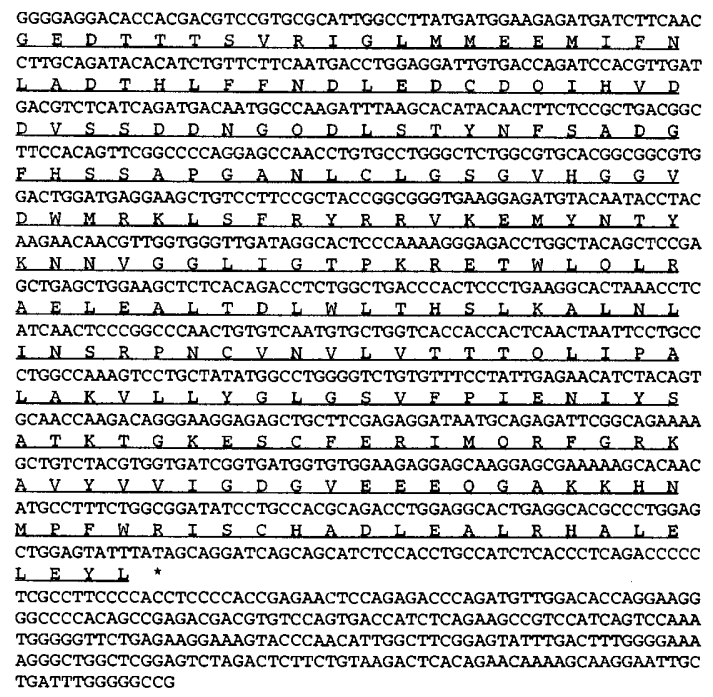

B

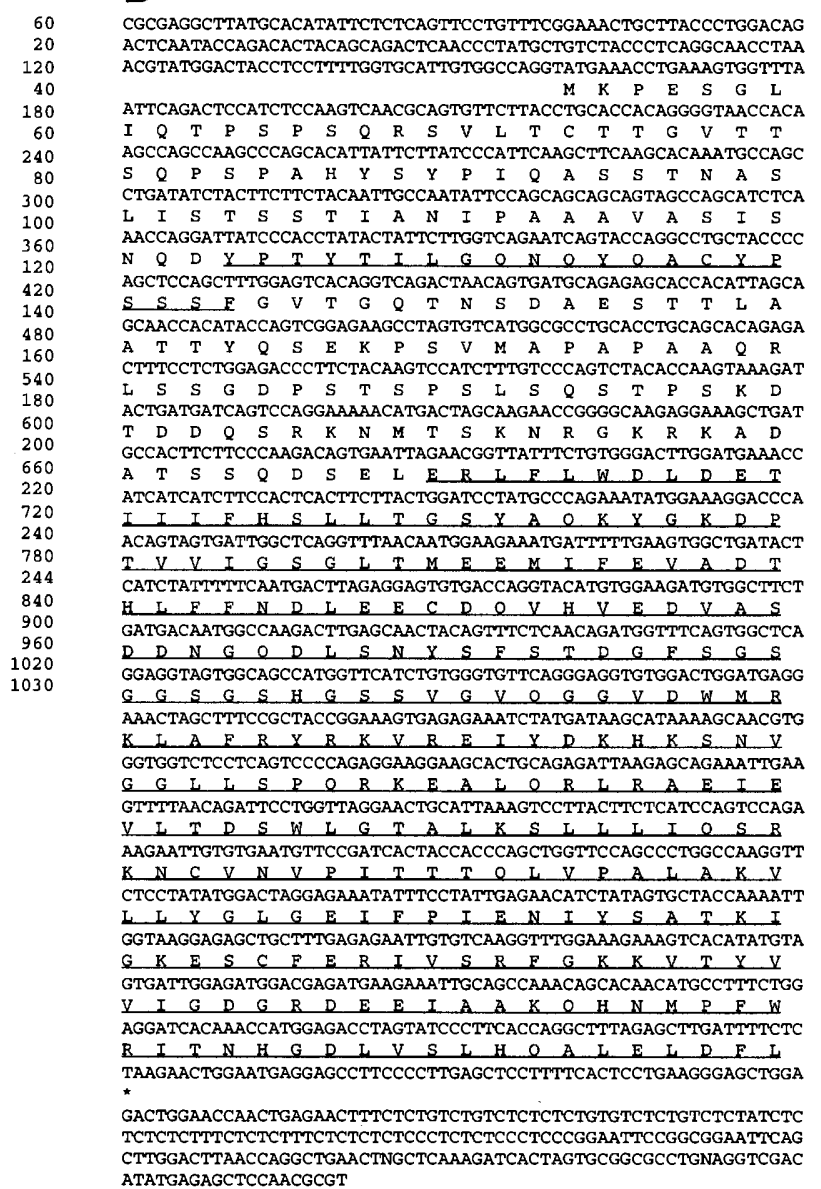

60

180

240

300
47

360

67
420

87

480
107

540

127
600

147

660
167

720

187

780
207

840

227

900
247

960

267

1020
287

1080

307

1140
327

1200

$\begin{array}{r}347 \\ \hline\end{array}$

1260

367
1320

1320
387

387
1380

407

1440
427

450
1500

447

1560

1620

1680
1740

1760

Figure 1 Sequence of human homologs of eya. DNA and predicted amino acid sequences of human eya homologs, EYA2 $(A)$ and $E Y A 3(B)$. The EYA2 clone has an incomplete ORF that stops within the large conserved domain ED1 (Eya homology domain 1). The EYA3 clone is translated from the first upstream methionine. The conserved domains ED1 and ED 2 (Eya homology domain 2) are underlined in the predicted protein sequences; ED1 is the large carboxy-terminal domain. GenBank accession nos. are U81601 for EYA2 and U81602 for EYA3.

other data (below) suggest that there may be alternative splicing at the $5^{\prime}$ end of the EYA3/Eya3 class of clones.

The longest mouse Eya2 clone predicts a $52-\mathrm{kD}$ protein of 473 amino acids, pI 5.5, assuming that the methionine at nucleotide position 166 is the initiation codon (Fig. 2A). The sequence around this potential translation initiation site (CAGCCATGG) shows strong consensus to the Kozak initiation sequence (CCA/GCCAUGG; Kozak 1981, 1984). The sequence upstream of this methionine, however, remains in-frame; possibly, there is an initiation methionine farther upstream. The largest ORF in the mouse Eya 3 clone predicts a 510 -amino-acid protein of $56 \mathrm{kD}$, pI 4.9 , assuming that the first methionine is the initiation site (Fig. 2B). The sequence around

Figure $2(A, B)$ Sequence of mouse homologs of eya. DNA and predicted amino acid sequences of mouse homologs Eya2 $(A)$ and Eya3 $(B)$. Both are translated from the farthest upstream in-frame methionine. Eya2 remains in-frame $5^{\prime}$ to the indicated start site, and therefore may predict a protein that is longer than indicated from the clones in hand. The conserved domains ED1 and ED2 are underlined in the predicted protein sequences; the ED1 domain is the large carboxy-terminal domain. GenBank accession nos. are U81603 for Eya2 and U81604 for Eya3. $(C, D)$ Comparison between human and mouse Eya homologs. Comparison of the amino acid sequences of the proteins predicted from human EYA2 and mouse Eya2 (C) and from human EYA3 and mouse Eya3 (D). The human and mouse Eya 2 sequences are $96 \%$ identical over the regions shown; the human and mouse Eya 3 clones are $95 \%$ identical over the carboxy-terminal 407 amino acids but diverge at their amino termini. Amino acid identities are boxed in black; similarities are shaded. 
A

GCACGAGCACCCAGCCTCGCAACAAGCAGTGACTGGAGCGAGCACGGTGCTGCCGTGGGG ACGCTGAGTGACAGGGAAGGCATCGCCAAATCAGCGGCTCTGAGTGTGCCTCAGCTCTTT GTGAAGTCTCATCCACGTGTCCCTCCTGGTCAGTCCTCCACAGCCATGGCGGCCTATGGC CAGACACAGTACAGCACAGGCATTCAGCAGGCACCACCCTATACAGCGTACCCAACTCCO

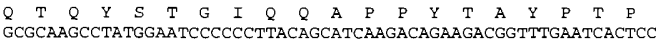
CCCAGCCAGAGCGGGTTCCTGAGCTATGGACCGAGCTTCAGCACCGCGCCTGCTGGACAG

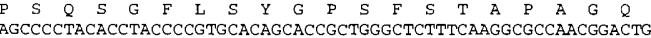

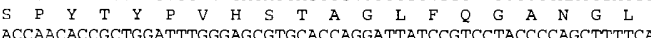

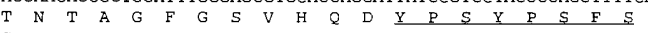
CAGAACCAGTACCCCCAGTATTTCAGCCCATCATACAACCCGCCCTACGTCCCTGCCAGC

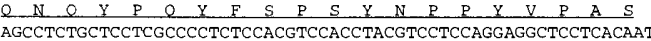

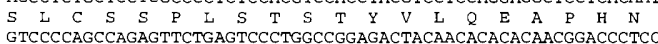

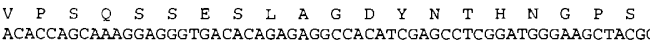
$\begin{array}{llllllllllllllllllll}T & P & A & K & E & G & D & T & E & R & P & H & R & A & S & D & G & K & L & R\end{array}$ GGCCGGTCAAAGAGAAATAGTGACCCTTCCCCAGCAGGAGACAATGAAATCGAGCGCGTG $\begin{array}{lllllllllllllllllll}G & R & S & K & R & N & S & D & P & S & P & A & G & D & N & E & I & E & R \\ \text { TTCGTCTGGGACCTGGACGAGACAATCATTATCTTCCACTCCCTGCTCACAGGGACGTTT }\end{array}$

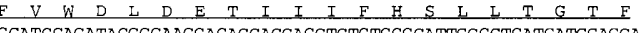
GCATCCAGATACGGGAAGGACACCACGACGTCTGTGCGCATTGGCCTGATGATGGAGGA

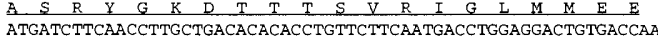

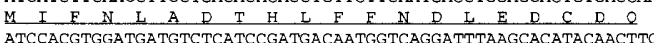

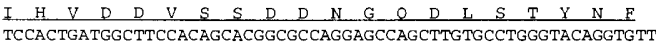

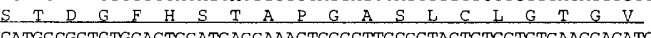
I G G V D U M R K U A $R$ Y $B$ Y TACAACACCTACCGCAACAACGTGGGTGGCTTGATAGGTGCTCCCAAAAGAGAGACCTG

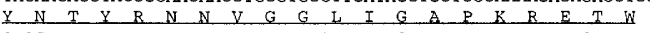
CTGCAGCTGCGCGCCGAGCTGGAGGCCCTGACTGACCTCTGGCTCACCCACTCCCTGAAA \begin{tabular}{llllllllllllllllllll}
$L$ & $O$ & $L$ & $R$ & $A$ & $E$ & $L$ & $E$ & $A$ & $L$ & $T$ & $D$ & $L$ & $W$ & $L$ & $T$ & $H$ & $S$ & $L$ & $K$ \\
\hline
\end{tabular} A

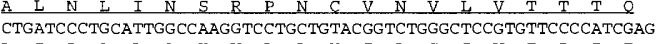

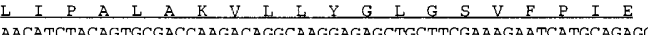

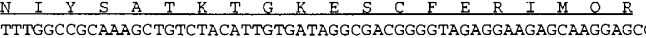

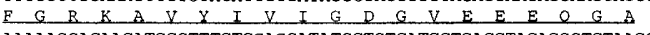
AAAAAGCACAACATGCCTTTCTGGAGGATATCCTGTCATGCTGACCTAGAGGCTCTAAG CATCCCCT

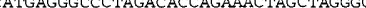
TCCTATACCTAGGGGACATGTTCCACGGCCATTTCAGAAGTGTCTTCTTCCCTTGGGGAC GGAGAGTCGGACTCTGATATGAAACCCAGAGACCCAAATGAGGGCGCCCCAGCTCGTCTI GTTCTCCTTTTTAATTATGGACTGCACCCGTTACTCCCATTACCCACGGGTCCCTTGTC GTTTCTCGGCTGTAGGTCTGGTTTCGGGTGGTGAGTACCCAGTITGTGTTTCAGGGACA TCATTCTCTGGGCGTTGGCGAGGGAGGTGGTGTGGGGCTGTGGGGAAGCCCGATGTCATG TGGACAGTGTGCGTGCCTTATGCCGCCATCTTGTGTTGTAGGAGAGGGGAACTCTGGGA GGCAAGGGTACTCGGCATGATGGATA.AAGGCATTCAATAAAAACCACGTTTACATTTTA AGTTCCCAAARTAATAAAAATCCATTTTGAACTTGTAAAAAAAAAAAAAAAAAAA

C

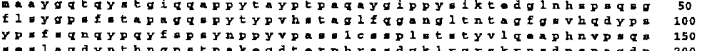

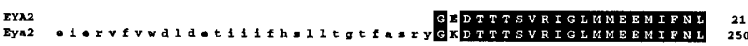

RrA
Braz

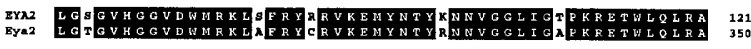

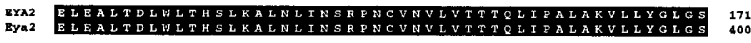

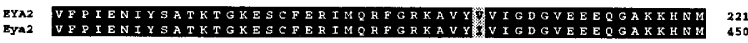

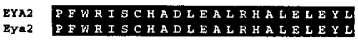

$\mathrm{D}$

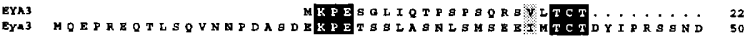

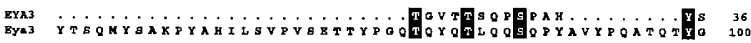

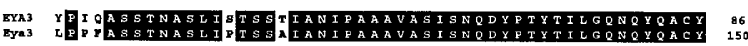

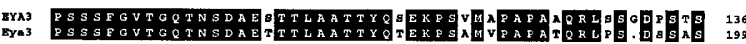

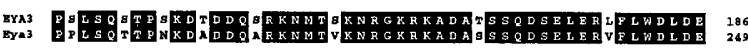

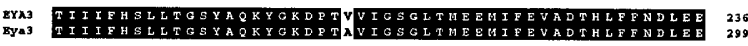

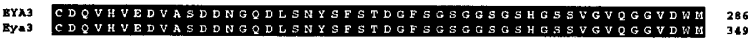

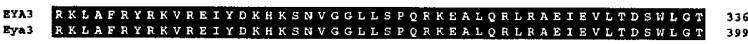

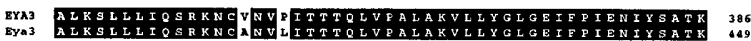

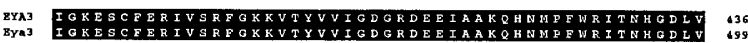

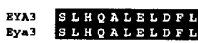

B

DCACGAGCCACTCTGTTGGTTCCAGAGTGGGTCCGGAATCGTCCCAAGTCGTGCTGGGC AGTGTCTGCAGCCGTCTGTTTG TGAAGACCGCAGAGACCTGCAATTCAGTCCCCTAAGT TGGCGTTATGCAAAGTCCTCATGGAAGAAGAGCAAGACCTACCAGAGCAACCGTTTCTA AGAGAACAGACTTTAAGTCAAGTAAACAACCCAGATGCCAGTGATGAGAAGCCTGAGACA

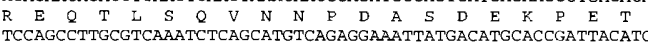

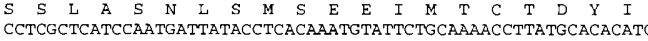

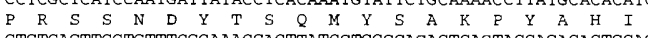
\begin{tabular}{lllllllllll} 
& $T$ & $Y$ & $P$ & $G$ & $Q$ & $T$ & $Y$ & $Y$ & $Q$ & $T$ \\
\hline
\end{tabular} CAATCTCAACCCTACGCTGTCTACCCTCAGGCAACCCAAACTTACGGACTACCTCCTTTC

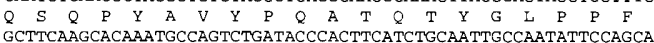

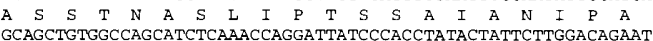
GCAGCTGTGGCCAGCATCTCAAACCAGGATTATCCCACCTATACTATTCTTGGACAGAA

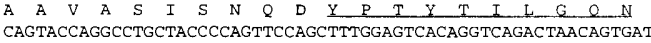

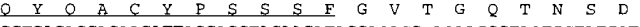

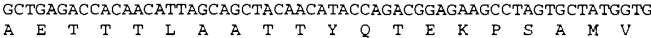

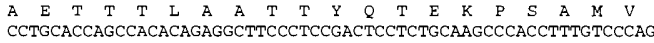

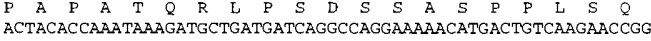

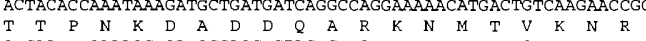

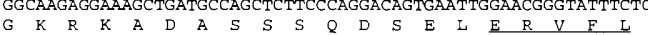
TGGGACTTGGACGAAACCATCATCATCTTTCATTCCCTTCTCACTGGATCCTATGCTCAG

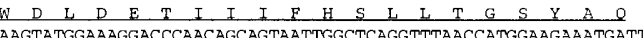

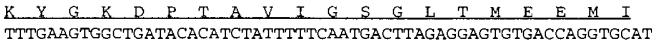

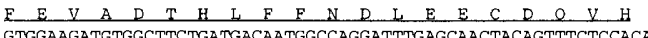

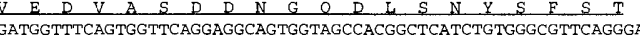
$\begin{array}{llllllllllllllllll}D & G & F & S & S & G & G & S & G & S & H & G & S & S & G & G & Q & G\end{array}$ GGTGTGGACTGGATGAGGAAACTGGCCTTTCGCTACCGAAAAGTGAGGGAAATCTACGAC \begin{tabular}{llllllllllllllllllll}
$G$ & $V$ & $D$ & $W$ & $M$ & $R$ & $K$ & $L$ & $A$ & $F$ & $R$ & $Y$ & $R$ & $K$ & $V$ & $R$ & $E$ & $I$ & $Y$ & $D$ \\
\hline
\end{tabular}

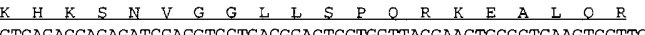
CTCAGAGCAGAGATCGAGGTGCTGACGGACTCCTGGTTAGGAACTGCGCTCAAGTCCTTE \begin{tabular}{llllllllllllllllllll}
$L$ & $R$ & $A$ & $E$ & $I$ & $E$ & $V$ & $L$ & $T$ & $D$ & $S$ & $W$ & $L$ & $G$ & $T$ & $A$ & $L$ & $K$ & $S$ & $L$ \\
\hline CTTCTCATCCAGTCTCGAAAGAACTGTGCGAATGTTCTGATCACTACCACGCAGTTGGTI &
\end{tabular} \begin{tabular}{llllllllllllllllllll}
$L$ & $L$ & $I$ & $O$ & $S$ & $R$ & $K$ & $N$ & $C$ & $A$ & $N$ & $V$ & $L$ & $I$ & $T$ & $T$ & $T$ & $O$ & $L$ & $V$ \\
\hline
\end{tabular} CCAGCCCTGGCCAAGGTTCTCCTGTATGGACTAGGAGAGATATTTCCTATTGAAAACA

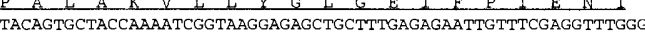
AAAAAAGTCACATATGTAGTGATTGGAGATGGACGAGATGAAGAAATTGCAGCCAAGCAG

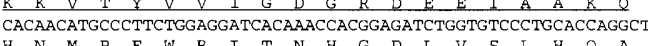

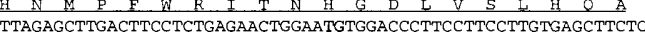

TTTACCTCCAACAGGAGCCAGAAGCCAAAACCCTCTGAGCCCCTTCTCTCCTGTCTGTCT GTCGGGTCTCAGTGCCCCCTCCCCCTTCTCTTCTGCTCTTTCTCCCTCCATGAAATGCTG GCGAGAACACAATCAGAACCAACAACTGCAGTTATTCTGAGTGAGCTGCAGCCCATGTCC TTTTTCTGTTTCAATTGAAAAAGGAAGAACAAGAAAAGCCGATGCTTGGGGCACAGTTGT ACTCTTGCTGCATGACGGACTGACCGGGAGCTGCTCCTGATGTTGTGGATGGAAACTGTC CCCTTGAGATCGTCTTCTGGTCTCTTAGCTATAGAATGTCTCTCCCAGTGAATGGGTATG TTATTTTTATAGGTGAGGGTCTGGTCTCTACAGCAGCCTCCCCCACTTTTCTATGAAGAA AGCCGTGTGTAAAAGTTTCCGTGACAGTAGTAATGGAAATATCTAAAATACCTCCGTGACT GGACAGGACAGAGGCTCCAGTGCTCTGCTCCCTGGGGAGGCTCCTTGCCATCTACAAGGC CCTGGTCACTTACTTTAGACTGTGGCATCTCCCATCTTGTAAGCCTCGGTCTCCTTGGCA GTCCTCCTTCATCTGTAACTAAATGGCACAACCCTGGAAACTCCTTCCCATAGACAATAA ATCTAAGCCTTTAGCTTGTACTTCAGAAGTTCTGTCCCAGGAGGCTCAGCCTCCAGACTG GAGAGAAGGGCTCAGATTTCTTAGGACTTTCACCTCGTGCTCCTGCAGAGCAGTACCTGT TCCCAGCAGATGCTTCCTCTGTGGTCGGCTCAGTCGTCAGATGCTGGCCTCACGTCCTGT CCTCACAGCTACCTGTCTCTGTGCAGCTCTGGCCAGCCCCTACCCACTCATTGCAAGTCA GAAAGGCCAAGGGGGCAGGCTCTAGCTGCCTCCTTCACCTGCACTTACATGCGGTGATCC CACCTTGTATTTATACAGATCTCTGCCTACGAGTGGAGAGCAGAGAGCTAGAGTCAAAAC CCATGAaATATCCCACICCTMGA TCCACGMG FTCTTGACTTAAGTGCTTAAAAATACAATCCTGAGCTAGGTGCAAGAGCACCTCTCAAG GTTTTGCTTTATTMCTTTTTGTTTGGGTTTTTTGTTCTTTTTACGGCACTATCCC TTCAGGACCAATGTCGGTGCCTGTTTAATTGGGTTAAACGTCCCTAGAGTTGAGGAAATG TGCAGCCTTGTTCTTCAGGAGGTCCTTGACCAACCCCTAGCTATAGTCACTGTCCGCACC GATACCACAGCCCAGAGGCAAAATTCCTCTTCATGCTAGGCTACCAGATAACGACAGAAA GGCTGACACCACCTTCAGAACTGTTGGACACTTCTGAGTACTTCTGAGACCAGCAGTTGA GGAAGGGGCTCAGACAGGAGGTGAGGTTTGTGTCT TGGCCATTCATTTGGCTTTGTTGI AGGTACTGGGTGGGAGCAAGGAGCTAGAGTCTAAGTCAGACCCTCCTAGGCTGCCATTAG CACCACACCGCTACCCTAGTCTGGCCAGCCTTGCTTGGTGGTCCAACCTGGGAAACTCCA GGATGAGTTTPACITCCAAGTCTCAG TCCTTCCCTCATTAACAGTGGAGTTTCTTGTGC

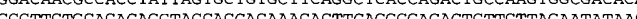
CCCTICTCACACAGGACACCACAACACTCAGGGGGACTCIICTTAGAATATAAC AGGTAATCCCTTCCTCTTGCCCTGACTGGAGAGGCAAGGAGG GMTGAGCTGAGTG GGGACTGCTGCCTTTGTGCTGACTCCCTCCCTCCCTCCCTCC 


\section{ZIMMERMAN ET AL.}

this potential translation site CCAAGATGC shows consensus to the Kozak initiation sequence. The longest human EYA3 clone, which differs from mouse Eya3 clones at its most amino-terminal portion (Figs. 1B and 2C), predicts a protein of 447 amino acids of $49 \mathrm{kD}$, with a pI of 5.5 .

Comparison of the predicted protein sequences of the vertebrate clones to the predicted fly Eya protein reveals several domains of homology (Fig. 3). The largest domain is the carboxy-terminal portion of the protein, referred to as ED1 (for Eya homology domain 1), which shows $53 \%$ identity over 271 amino acids of all the vertebrate and fly clones. Comparison of the vertebrate clones individually to the fly sequence in this carboxy-terminal region shows EYA2 to be $64 \%$ identical and the mouse Eya 2 class to be $67 \%$ identical, whereas the human EYA3 and mouse Eya3 classes are $62 \%$ identical to the predicted fly protein sequence. Within the amino-terminal domain, the sequences show little conservation. A short region of weak homology, ED2 (Eya homology domain 2), occurs between the vertebrate and fly sequences in the amino terminus. In this domain, a run of spaced tyrosine residues is conserved (Fig. 3C); the homology in this region to the Eya 2 homolog is greater and longer $(10 / 28$ amino acids for $36 \%$ identity) than with the EYA3/ Eya3 homologs (5/21 amino acids for $24 \%$ identity). Because of the low level of homology, the significance of this region is unclear. In addition, the fly sequence has a weak PEST protein degradation sequence; in a similar location, the mouse Eya2 sequence has a region that may serve as a weak PEST site (Fig. 3A). The fly sequence has a run of basic charge between the ED1 and ED2 domains (see Fig. 3A; Bonini et al. 1993). Although this sequence is not strongly conserved, a short cluster of basic charge occurs in a similar location in both Eya2 and the EYA3/Eya3 homologs (Fig. 3A). The fly Eya protein sequence has a consensus nuclear localization signal at the amino terminus, and the protein is nuclear by immunocytochemistry (Bonini et al. 1993). However, none of the vertebrate sequences show a motif indicative of a nuclear localization sequence (Chelsky et al. 1989; Dingwell and Laskey 1991); possibly, the cluster of conserved basic charge noted above, or a second region of basic charge within the ED1 conserved domain (in the fly, amino acids 600-629) serves this purpose.

\section{The Mouse Eya Homologs Are Expressed in the Eye}

We addressed expression pattern of the vertebrate homologs using the mouse clones to probe Northern blots of poly $(\mathrm{A})^{+}$RNA isolated from various mouse tissues, including the eye. Unique fragments in the 3'-untranslated regions for the respective genes were used as probes. Both Eya2 and Eya 3 were strongly expressed in poly $(\mathrm{A})^{+} \mathrm{RNA}$ isolated from adult mouse eye (Figs. 4A,D). For Eya2, a similar sized transcript of $2.4 \mathrm{~kb}$ was expressed in the eye as in other tissues of the animal. The Eya3 probe, however, detected multiple transcripts in the eye-one strongly expressed transcript of $5.5 \mathrm{~kb}$ and three additional transcripts of $4.4,2.5$, and $1.9 \mathrm{~kb}$.

In other tissues and during development the Eya2 gene showed a more restricted pattern of expression compared to the Eya3 gene. During embryonic development, the Eya2 gene was first strongly expressed at 11 days and then showed a gradual reduction in expression to 17 days (Fig. 4B). In contrast, the Eya3 gene was expressed at roughly similar levels during all stages of mouse embryonic development, with only the largest $5.5-\mathrm{kb}$ transcript be-

Figure $3(A)$ Conserved domains of eya homologs of human, mouse, and fly. Schematic representation of domains of homology of the fly, mouse, and human clones. Select additional features are indicated, including the consensus nuclear localization signal (NLS) of the fly sequence and a domain enriched in amino acids of basic charge $(++)$. Percent amino acid identity with the fly sequence is indicated for ED1 and ED2 for each homolog. In the fly sequence of the type I CDNA, the NLS runs from amino acid 18 to 23 , the opa repeat from amino acid 40 to 62 , and ED2 from amino acid 326 to 354 , potential PEST sequence from amino acid 373 to 388 , basic domain 1 from amino acid 449 to 471 , ED1 from amino acid 487 to 760 . The human EYA2 sequence is incomplete at the $5^{\prime}$ end, and the incomplete ORF is indicated with an asterisk $\left(^{*}\right)$. In mouse Eya2, ED2 runs from amino acid 98 to 125, the potential PEST sequence from amino acid 144 to 161, the short charge cluster from amino acid 183 to 190, and ED1 from amino acid 203 to 473. In human EYA3, ED2 runs from amino acid 71 to 91, short cluster of basic charge from amino acid 159 to 165, and ED1 from amino acid 177 to 447. In mouse Eya3, ED2 runs from amino acid 135 to 155, the basic charge cluster from amino acid 222 to 228, and ED1 from amino acid 240 to 510 . $(B, C)$ Sequence lineups of ED1 $(B)$ and ED2 (C) of fly, human, and mouse homologs. Amino acid identities are boxed in black; conservative changes are shaded. The consensus sequences for ED1 were derived in relation to the fly sequence. Amino acid similarities were defined with the default symbol comparison table based on the Dayhoff PAM-250 matrix. By this program, the following amino acids are considered similar: $F, Y ; L, M ; I, V ; E, D$. 
A Fly Eya $\mathrm{NLS}$ opa Eya Domain 2 PEST Domain 1

$64 \%$
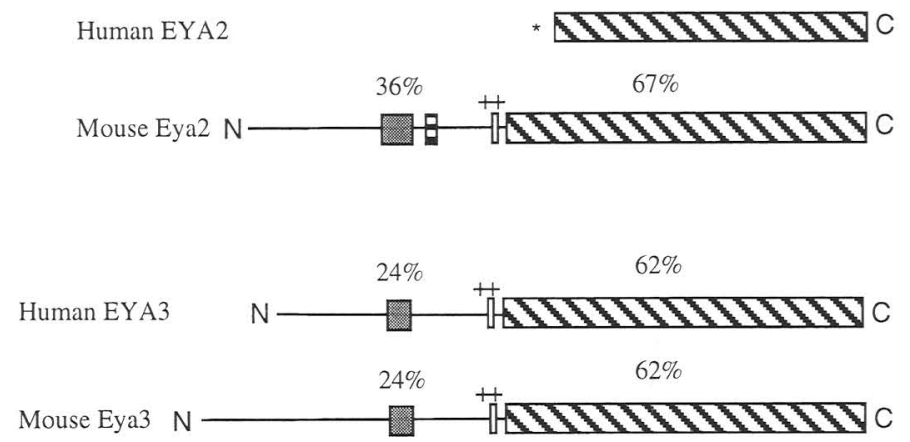

B

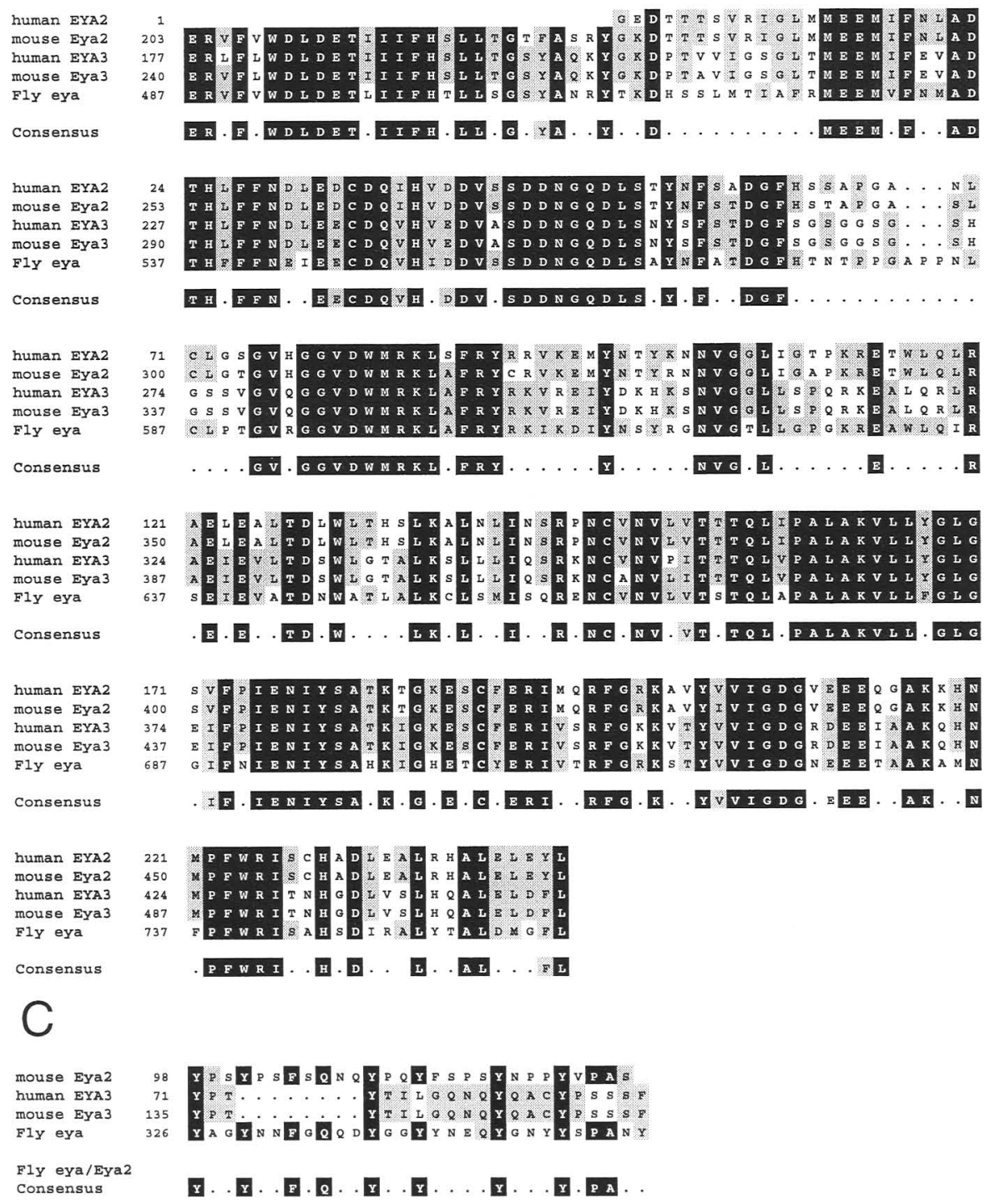

Figure 3 (See facing page for legend.) 


\section{ZIMMERMAN ET AL.}

ing expressed to appreciable levels (Fig. 4E). In adult tissues, the 5.5-kb transcript of the Eya3 gene was expressed in all tissues tested except spleen, whereas Eya2 expression was detectable only in lung (Fig. 4 $\mathrm{C}, \mathrm{F})$. We also determined that Eya2 was expressed in thymus and uterus (data not shown).

\section{Chromosomal Mapping of the Genes}

A chromosomal location of the mouse Eya2 gene was originally established in the Jackson Laboratory Backcross DNA Panel-a community genetic mapping resource (Rowe et al. 1994). Segregation analysis of backcross progeny placed the Eya2 gene to the distal portion of mouse chromosome 2 . No recombinants were detected between Eya2 and the Iapt15 (intercisternal A particle tumor-specific-15) gene in 91 animals typed in common, whereas the same cross placed the Eya2 gene $6.3 \mathrm{cM}$ proximal to the Gnas gene (upper 95\% confidence limit). We compared the map location of Eya2 with composite mouse linkage maps that report the map location of many uncloned mouse mutations (provided from the mouse genome database, a computerized database maintained at the Jackson Laboratory). Eya2 maps in a region of the composite map that contains the eye mutation blind-sterile [bs (Varnum 1983)]. The $b s$ locus has been mapped $1.7 \pm 1.2 \mathrm{cM}$ proximal to Emv13/Emv15 loci (Spence et al. 1992). Because the Emv13/Emv15 loci were mapped previously in another large interspecific backcross, typed for $>2200$ loci (Copeland and Jenkins 1991), Eya2 and Eya3 were mapped in this backcross. The mapping results confirmed that Eya2 is located in the distal region of chromosome 2 and showed that the Eya3 gene maps to the distal region of chromosome 4 (Fig. 5). Moreover, this set of mapping data determined that $b s$ is not a candidate for a mutation in $E y a 2$, as bs has been located proximal to the ectopic murine provirus-15 (Emv15) gene by interspecific backcross mapping (Spence et al. 1992) while Eya2 has been mapped distal to this marker. No recombinants were detected between the Eya3 gene and the $F g r$ genetic locus in 83 animals typed in common, suggesting that these loci are within $4.3 \mathrm{cM}$ of each other (upper 95\% confidence limit). There are no known eye-specific mutations that map in this chromosomal region.

We performed fluorescence in situ hybridization (FISH) with a P1 probe containing the human EYA3 gene and demonstrated that the EYA3 gene hybridized to the terminal band of chromosome 1 (1p36) in all cells examined (Fig. 6). This is the region suggested from the mouse Eya3 mapping localiza- tion, based on synteny between mouse and human chromosomes (Fig. 5). The human EYA2 gene has been localized to human chromosome 20 (20p13.1; Banfi et al. 1996), which is the region suggested by the mapping of the mouse Eya2 gene (Fig. 5).

\section{DISCUSSION}

\section{Eya Gene Family}

We report the isolation and initial characterization of two vertebrate genes related in sequence to the Drosophila eya gene. This analysis has revealed striking features of the eya protein sequence that are conserved in vertebrates. The largest domain of homology, ED1, spans $\sim 270$ amino acids and covers the carboxy-terminal portion of the predicted proteins. The degree of homology over this long stretch of amino acids $-53 \%$ over all vertebrate and fly protein sequences analyzed here-suggests that this domain is of special importance to the function of the gene products. Within this region are short amino acid runs of exceptionally high conservation (see consensus sequence, Fig. 3B); however, there are no previously defined motifs that speak to biochemical function. Rather, this domain defines a new domain of conservation. A second, smaller amino-terminal domain, ED2, of $\sim 30$ amino acids shows conservation for fly and Eya 2 of a spaced run of tyrosine amino acids. Other features common to the vertebrate and fly gene products include a region of enriched basic charge just amino-terminal to the large ED1 domain, and, in the Eya2 homolog, a possible PEST protein degradation sequence.

Overall, the vertebrate genes predict proteins with the most homologous domain limited to the carboxy-terminal portion of the protein. The amino-terminal region of the fly sequence, which contains numerous runs of repeated amino acids, may not be critical to function of the protein, with the exception of the short regions of homology noted. Alternatively, the amino terminus may contain important biological information that is not recognized as a conserved sequence, or is not conserved in the vertebrate homologs identified to date. In the latter case, we might anticipate the eventual identification of proteins with greater homology to the amino terminus. Notably the fly sequence has an opa triplet repeat at the amino terminus that is not present in any of the vertebrate sequences defined so far. Triplet repeats tend to be associated with genes important to nervous system function and development; expansion of such triplet repeats is associated with a number of human neurodegen- 


\section{VERTEBRATE HOMOLOGS OF DROSOPHILA EYES ABSENT}
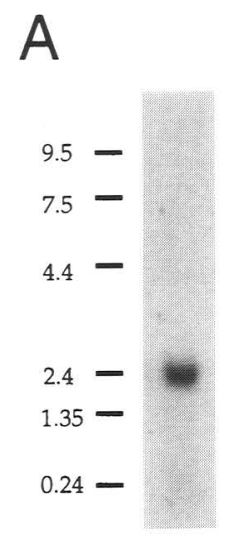

D

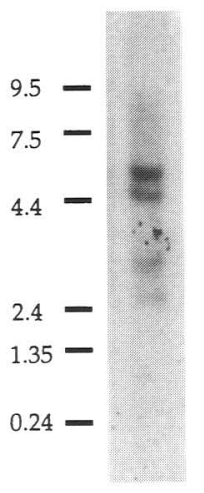

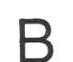
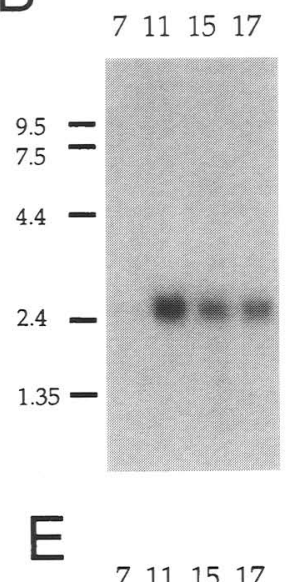

$\begin{array}{llll}7 & 11 & 15 & 17\end{array}$

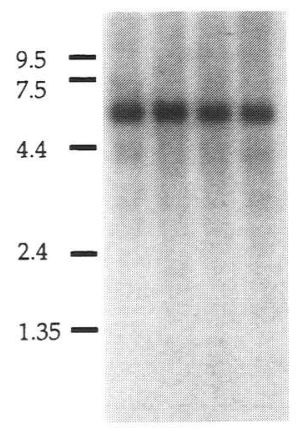

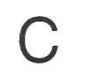

$9.5=$

$4.4-$

$2.4=$

$1.35-$
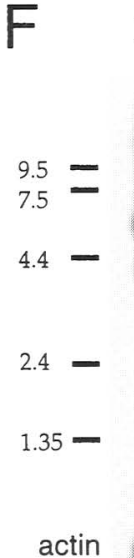

$\mathrm{H} \quad \mathrm{B} \quad \mathrm{S}$ L Li $\mathrm{M}$ K $\mathrm{T}$

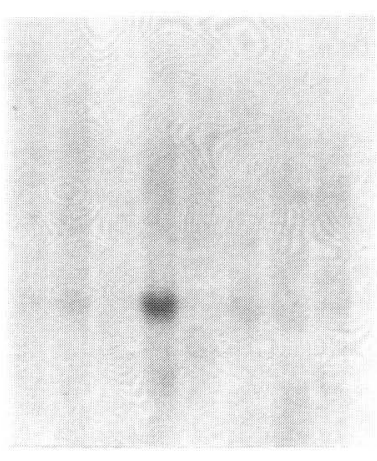

H B S L Li M K T

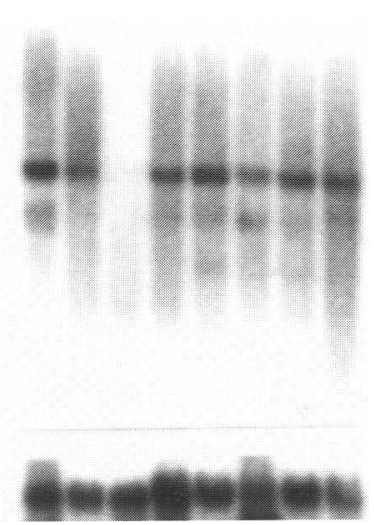

Figure 4 Expression by Northern analysis of the mouse Eya2 and Eya3 genes. Expression of Eya2 $(A)$ and Eya3 $(D)$ genes is shown in poly $(A)^{+}$RNA isolated from mouse adult eye. Expression of the Eya2 $(B)$ and Eya3 $(E)$ genes is shown at 7, 11, 15, and 17 days of mouse embryonic development. Expression of the Eya2 (C) and Eya3 $(F)$ genes is shown in adult tissues; the panel beneath $F$ is the same blot hybridized with a $\beta$-actin CDNA probe. Lanes are labeled as follows: $(\mathrm{H})$ Heart; (B) brain; (S) spleen; (L) lung; (Li) liver; (M) skeletal muscle; (K) kidney; (T) testis.

erative diseases (Ashley and Warren 1995; Karlin and Burge 1996). As mutation of the fly gene leads to cell death, at least in the eye (Bonini et al. 1993), it will be of interest to determine whether there may be an additional vertebrate eya homolog that contains this repeat or whether there are alternative splice products of the currently defined genes that contain a triplet repeat. The mouse Eya3 gene, for which we have defined a number of alternatively spliced products expressed in the eye in particular, is such a candidate. The mouse Eya 3 and human EYA3 genes, although highly similar over most of the predicted amino acid sequence, diverge significantly at their amino termini. Alternative splicing likely accounts for this variation, with the Eya 3 and EYA3 clones that we have defined representing different splice variants. Consistent with this, another mouse
Eya3 clone identified ( $\mathrm{Xu}$ et al. 1997) displays alternative splicing at the $5^{\prime}$ end compared to our Eya 3 clones. The isolation of additional splice forms, as well as analysis of genomic clones, will aid in defining the intron/exon structures of the vertebrate genes. In addition, analysis of mutations in the fly that alter specific subfunctions of the gene (Bonini et al. 1993; Leiserson et al. 1994) may reveal roles of the amino-terminal domain and potential regulatory elements, in addition to defining amino acids within the highly conserved domains that are critical for function.

The two mouse Eya genes show striking differences in expression during development and in adult tissue. The Eya2 gene is expressed in a rather restricted manner, being expressed strongly in select adult tissues and with a temporal pattern developmentally, whereas the Eya3 gene is expressed in a more widespread manner. This indicates that the genes are likely to be under distinct regulatory control, both during development and in the adult animal. These data are supported by tissue in situ analysis of the genes during mouse development, which also demonstrate widespread expression of the mouse Eya1 gene (Xu et al. 1997). All genes, however, are expressed in eye tissue during development (Xu et al. 1997), and at least Eya 2 and Eya 3 in the adult eye, with the Eya3 gene showing appreciable levels of expression of multiple transcripts (see Fig. 4). During development, the genes show complex tissue expression, especially in the nervous system, some of which is in overlapping tissues and other in adjacent tissues (Xu et al. 1997).

The distal region of mouse chromosome 2 is syntenic with the long arm of human chromosome 20 , whereas the distal part of chromosome 4 is syntenic with human chromosome 1 (see Figs. 5 and 6). The placement of Eya2 between the markers adeno- 

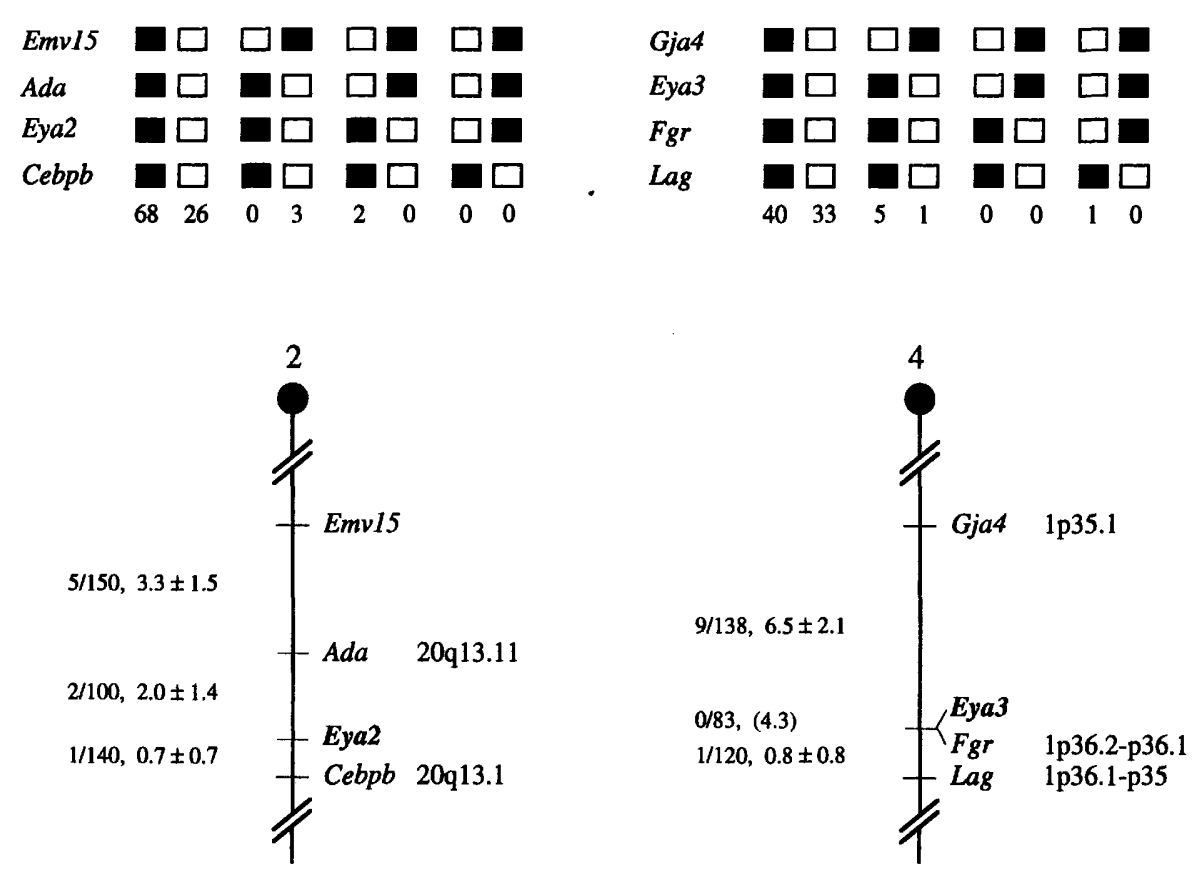

Figure 5 Chromosomal map positions of mouse Eya2 and Eya 3 in the Frederick interspecific backcross. Partial chromosome linkage maps showing the mouse chromosomal location of Eya 2 and Eya 3 as determined by interspecific backcross analysis. The segregation patterns of the two Eya genes and flanking genes are shown at the top. Each column represents the chromosome identified in the backcross progeny that was inherited from the $(\mathrm{C} 57 \mathrm{BL} / 6 \mathrm{~J} \times M$. spretus $) \mathrm{F}_{1}$ parent. ( $\square$ ) The presence of a $\mathrm{C} 57 \mathrm{BL} / 6 \mathrm{~J}$ allele; $(\square)$ the presence of a $M$. spretus allele. The number of offspring inheriting each type of chromosome is listed beneath each column. Although 99 mice were analyzed for every marker shown in the segregation analysis of Eya2, up to 150 mice were typed for some pairs of markers. Similarly, although 80 mice were analyzed for every marker shown in the segregation analysis of Eya3, up to 138 animals were typed for some pairs of markers. Partial chromosome linkage maps of chromosomes 2 and 4, indicating the location of Eya 2 and Eya 3 in relation to linked genes, are shown at the bottom. Recombination distances between loci ( $\mathrm{cM}$ ) are indicated to the left of the chromosome $( \pm$ S.E.); the positions of loci in human chromosomes are shown to the right. Where no recombinants were found between loci, the upper $95 \%$ confidence limit of the recombination distance is given in parentheses. References for the human map positions of loci cited in this study can be obtained from GDB (Genome Data Base; http://gdbwww.gdb.org/), a computerized database of human linkage information maintained by the William $\mathrm{H}$. Welch Medical Library of the Johns Hopkins University (Baltimore, MD).

sine deaminase (Ada) and CCAAT/enhancer binding protein $\beta(C e b p b)$ suggests that the human Eya2 gene resides on human chromosome 20q13.1; this has been shown to be the case using a human clone of the Eya2 class (Banfi et al. 1996). Similarly, the tight linkage between Eya3 and Fgr suggested that human Eya3 lies on human chromosome 1p36.2p36.1. FISH analysis presented in this paper confirms the localization to the terminal portion of human chromosome 1 . Several interesting genetic disorders have been located to these two chromosomal regions, although localization to the EYA2/Eya2 and EYA3/Eya3 genes has not yet been demonstrated. Although the mouse Eya2 homolog maps near the mouse eye mutation $b s$, and $b s$ has been speculated to be a mutation in the Eya2 gene (Banfi et al. 1996), our mapping data rule out this possibility.

\section{Evolution of Eya}

Based on the large and highly conserved ED1 domain, we have constructed a similarity tree defining the relatedness of the eya homologs currently identified (Fig. 7). The tree suggests that two gene duplication events occurred in vertebrates, one leading to the separation of the Eya3 branch from the Eya1 and Eya2 branch, and a second duplication event leading to the Eya1 and Eya2 genes. Furthermore, existence of a human homolog of the mouse Eya1 gene is predicted. The mammalian genes apparently duplicated about the time of the fly/mammalian divergence, as all vertebrate genes show a relatively similar degree of divergence from the fly gene. At this point in time, other Drosophila genes related in sequence to Eya have not been defined. One interpretation would be that multiple fly eya genes do not exist and that the divergence observed in vertebrates occurred subsequent to the fly/vertebrate split. It will be of interest to determine the extent to which functional properties assigned to the fly gene be assigned to members of the vertebrate Eya family, or whether the proteins have diverged, at least in part, in function. Our results also suggest the possibility that fly eya may be the founding member of an even larger class of vertebrate homologs, as Southern blot studies with the M306 probe are indicative of additional related genes. 


\section{VERTEBRATE HOMOLOGS OF DROSOPHILA EYES ABSENT}

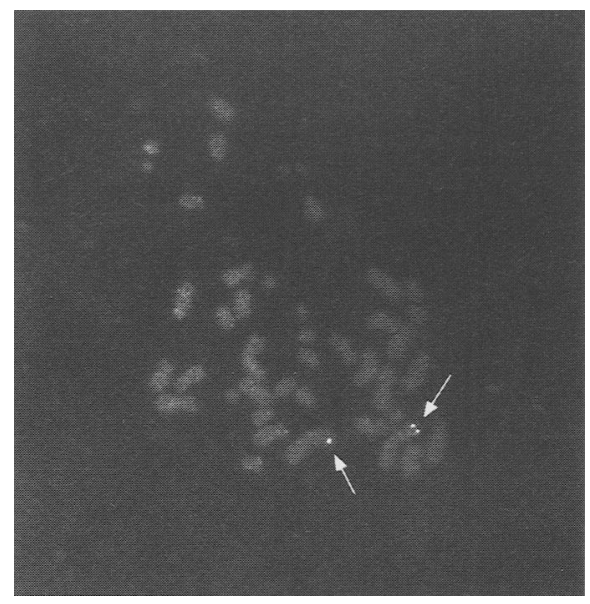

Figure 6 Localization of EYA3 to human chromosome 1. FISH of a $\mathrm{P} 1$ probe containing EYA3 on a metaphase spread from a normal individual. Signal is present on chromosome 1p36 on both homologs (arrows). Localization was also performed by doublelabeling with the P1 probe and a chromosome 1-specific $\alpha$-satellite probe (data not shown).

The biochemical mechanism of action of the fly Eya product has not yet been defined, although the protein is known to be nuclear. Whereas the fly protein has a consensus nuclear localization sequence, none of the vertebrate clones shows conservation of this sequence. A fundamental feature of nuclear localization sequences is a basic charge (Dingwell and Laskey 1991), so possibly the region of basic charge amino-terminal to the ED1 domain, or other sites of basic charge within the predicted proteins, subserves this purpose. Alternatively, the proteins may be carried into the nucleus through association with other proteins that have more conventional nuclear localization signals. Divergence of the proteins at the amino-terminus may therefore indicate functions related to subcellular localization, among other possibilities. Nevertheless, defining whether the vertebrate proteins are localized to the nucleus may be crucial for addressing potential conservation of biological function of the genes. Moreover, although the vertebrate proteins show striking homology to the fly protein, especially in the carboxyl terminus, it will be of interest to address whether the vertebrate homologs are similar enough in sequence to substitute biologically for their fly counterpart. Such a test will address the degree to which the biological pathway of eya gene activity is conserved from flies to vertebrates, potentially addressing fundamental conservation of gene order and function in the Pax-6/eyeless pathway of eye devel- opment. The identification of interacting proteins of known function, as well as analysis of the role of the eya family of genes in the eye and other tissues of flies and vertebrates, will lead toward an understanding of the biological role of these genes.

\section{Evolutionary Pathway of Eye Development}

The fly eya gene has a critical role in development of the eye, with selective loss of gene function in the eye leading to complete loss of this structure (Bonini et al. 1993). Striking conservation of fly and verte-

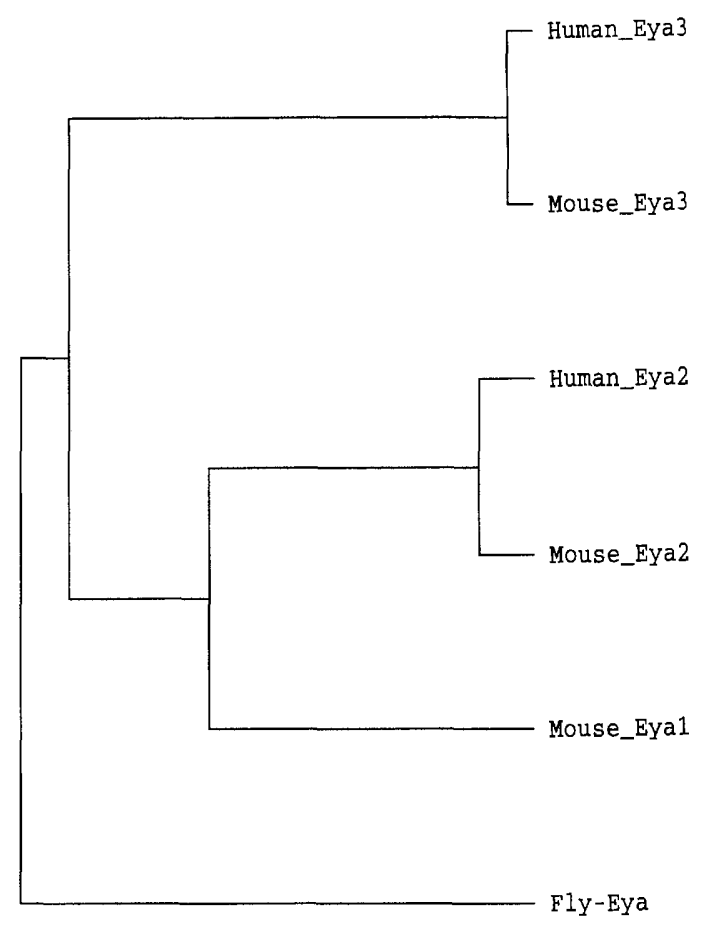

Figure 7 Similarity tree of eya family homologs. The tree was constructed from sequence alignment with the PILEUP Program (GCG; University of Wisconsin). Only the 271 amino acid ED1 domain, which is 274 amino acids in the fly, were used for the alignment. For the human EYA2 homolog, which is incomplete for the amino-terminal 27 amino acids of this domain, we completed that part of the sequence using the homologous region of the mouse Eya2 homolog: we reasoned that the human EYA3 and mouse Eya3 genes predicted proteins are $100 \%$ identical over this sequence region; therefore, the human EYA2 and mouse Eya2 genes are likely to be identical as well. However, using the incomplete sequence of human EYA2 for construction of the tree results in a similarity tree that is not significantly altered. The mouse Eya1 sequence is from GenBank accession no. U61110. A human EYA1 gene has not yet been characterized; however, the tree predicts that it exists. 


\section{ZIMMERMAN ET AL.}

brate genes expressed and functioning in the eye is seen with the eyeless/Pax-6 paired-box and homeobox gene (Quiring et al. 1994; Halder et al. 1995), and the Six-3 homeobox gene, defined by the fly mutant sine oculis (Cheyette et al. 1994; Serikaku and O'Tousa 1994; Oliver et al. 1995). The Eya2 and Eya 3 genes are both expressed in the mouse eye in the adult (see Fig. 4), and other studies have shown that all three mouse Eya genes are expressed in the developing eye (Xu et al. 1997). Thus, we anticipate that the vertebrate Eya genes will function in eye development, as their counterpart does in the fly.

These three genes-eyeless, sine oculis, and eyashare the property of being essential early in Drosophila eye development, with expression prior to the first signs of neural differentiation (Bonini et al. 1993; Cheyette et al. 1994; Quiring et al. 1994; Serikaku and O'Tousa 1994). This raises the possibility that a fundamental set of genes (eyeless/Pax-6, sine oculis/Six-3, and Eya among them) functions across a remarkably large evolutionary distance in eye differentiation. It has been proposed recently that the eye of both vertebrates and invertebrates, despite dramatic structural differences, evolved from a common ancestor with a primitive eye (Halder et al. 1995; Gehring 1996). The alternative and longstanding hypothesis is that the fly and vertebrate eye evolved independently (see Dickinson and Seger 1996). Without necessarily distinguishing between these two hypotheses, in this report we add weight to the argument that the fly and vertebrate eye arose because of the use of a common set of genes. With respect to this issue, both Pax-6 and Six-3 genes contain motifs indicating that they function as transcription factors, and, at least in the fly, the Eya protein shows nuclear localization. Will potential targets of these genes be conserved from flies to vertebrates? For the Eya gene family, some aspects of fly eya gene regulation are remarkably specific to expression and function of the gene in the eye (Leiserson et al. 1994); it will be of interest to determine whether related regulatory elements are found in the Eya vertebrate genes. Toward this end, studies in the mouse indicate that expression of Eya genes is reduced in Pax-6 mutant mice (Xu et al. 1997).

A related and important issue is that these genes have functions in addition to eye development, as mutants have phenotypes in addition to loss of the eye and gene expression is not limited to the eye (Bonini et al. 1993; Cheyette et al. 1994; Quiring et al. 1994; Serikaku and O'Tousa 1994). For example, mutations of eya in the fly can be embryonic lethal, as well as show adult female sterility in select combinations of alleles (Nüsslein-Volhard et al. 1984;
Bonini et al. 1993). Expression of the vertebrate Eya genes in tissues other than the eye was thus anticipated and confirmed by our study. Pax-6 is present in organisms including Caenorhabditis elegans, which have no photoreceptor cells (Chisholm and Horvitz 1995; Zhang and Emmons 1995). These observations therefore raise the questions, is there a fundamental function of these genes in animal patterning, and how does their function lead to the differentiation of an eye in select cells of some animals, but not in other cells nor in other animals? This property of selective eye formation is true even for the Pax-6/eyeless gene, which can direct ectopic eye formation when aberrantly expressed.

One hypothesis is that the pathway involving these genes arose specifically for eye development. An alternative-and we would argue, more likelyhypothesis, however, is that these genes may be part of a genetic network that underlies a more fundamental signaling process than eye development itself. During evolution, a genetic circuit using these genes is likely to have arisen in a primitive ancestor of both flies and vertebrates-the gene network itself being more ancient than eye development. Subsequently, the entire circuit became co-opted into eye development at least once. In theory, such a hypothesis does not distinguish between the vertebrate and fly eyes having evolved either from a common ancestor or independently, as the genetic circuit could have been co-opted into eye development multiple times. In view of these issues, it will be of interest to define in detail the genetic interactions among these genes, whether a circuit involving these genes is present in tissues other than the eye, and whether circuits involving these genes are present in all other organisms with eyes, and in those like C. elegans, which have no eyes.

\section{METHODS}

\section{Isolation of cDNA Clones}

Clone 1 ce06 (GenBank accession no. Z39529) was obtained from the Genexpress cDNA Program, Laboratoire Genethon, Evry, France. Primers were designed to the ends of the sequence available of this clone: a forward primer $5^{\prime}$ GACTGGATGAGGAAACTAGCTTTC-3', corresponding to amino acids DWMRKLAF, and a reverse primer $5^{\prime}$ GGTAGCACTATAGATGTTCTCAATAGG-3', corresponding to the amino acids PIENIYSAT, which fall within a conserved domain of EYA. The primers were used to amplify a predicted 306-bp product from mouse brain cDNA made by RT-PCR from mouse brain mRNA of stage E9.5 and to amplify a 306bp product from a human retinal library (Clontech). Amplification was performed by standard protocols using an annealing temperature of $55^{\circ} \mathrm{C}$, extension at $72^{\circ} \mathrm{C}$, for 35 cycles. The amplification product M306 was used to probe a mouse 


\section{VERTEBRATE HOMOLOGS OF DROSOPHILA EYES ABSENT}

brain cDNA library (Stratagene), and a mouse retinal cDNA library (American Type Culture Collection). The human product H306 was used to probe human retinal and human brain libraries (Clontech). Positive clones were amplified from phage and subcloned into pGEM-T (Promega), tested for cross-hybridization, mapped, and sequenced by automated sequencing using sequential primers. The mouse Eya 2 sequence is a composite of sequence derived from clones M27 and M30; the mouse Eya3 sequence from clone M25; the human EYA2 sequence from clones $\mathrm{H} 13$ and $\mathrm{H} 12$; and the human EYA3 sequence from clones 1 ce06 and HB1. The $\mathrm{H} 13$ and 1ce06 clones were fusion cDNAs with part of the sequence highly homologous to eya and part of the sequence completely divergent; the divergent parts of the sequences were removed in this analysis. For Southern hybridization, mouse genomic DNA of strain C57BL/6J was digested with BamHI, PstI, SalI, and $\mathrm{XbaI}$, run on a $7 \%$ agarose gel, and transferred to nitrocellulose following standard protocols. Blots were probed with radioactively labeled M306, Eya2 and Eya3 clones at $5 \times 10^{6} \mathrm{cpm} / \mathrm{ml}$, and exposed by PhosphorImager analysis.

\section{Sequence Analysis}

Sequence comparisons between fly and vertebrate ORFs were performed using the GCG software package, version 8 (Genetics Computer Group), with the program PILEUP, presented with Microsoft Excel highlighting of sequence identity and alignment (Haygood 1993). Amino acid similarities were defined with the default symbol comparison table based on the Dayhoff PAM-250 matrix. By this program, the following amino acids are considered similar: F ,Y; L, M; I, V; E, D. The ED2 domain was identified with the sequence alignment program MACAW (National Center for Biotechnology Information). The phylogenetic tree was generated using programs available from the GCG software package, from the PILEUP alignment.

\section{Northern Blot Analysis}

Northern blots with poly $(\mathrm{A})^{+}$RNA from various adult mouse tissues and different developmental stages of the mouse ( $2 \mu \mathrm{g}$ per lane on formaldehyde $/ 1.2 \%$ agarose gels) were purchased from Clontech. For mouse adult eye RNA, total RNA was isolated from adult female mouse eyes of the CF1 strain using Trizol reagent (GIBCO-BRL). Total RNA was stored as a precipitate until use in $100 \%$ ethanol at $-70^{\circ} \mathrm{C}$. Poly $(\mathrm{A})^{+}$RNA was isolated using oligo(dT) columns (Clontech) and stored as an ethanol precipitate until use. RNA ( $2 \mu \mathrm{g}$ per lane) was electrophoresed, using a formaldehyde/ $1 \%$ agarose gel in $1 \times \mathrm{TAE}$ buffer containing $0.6 \%$ formaldehyde, and transferred to positive charged nylon following standard procedures (Sambrook et al. 1989). For all probing, blots were prehybridized for $30 \mathrm{~min}$ at $68^{\circ} \mathrm{C}$ and hybridized for $1 \mathrm{hr}$ at the same temperature in ExpressHyb solution (Clontech). The probes used were 3' untranslated regions of clone M27 for the Eya2 gene and of clone M25 for the Eya3, and a human $\beta$-actin cDNA probe supplied by Clontech. Probes were labeled with $\left[\alpha{ }^{32} \mathrm{P}\right]$ dCTP using random hexamers following standard procedures (Sambrook et al. 1989). The blots were washed for $40 \mathrm{~min}$ in $2 \times$ SSC, $0.05 \%$ SDS, at room temperature followed by $40-60$ min in $0.1 \times \mathrm{SSC}, 0.1 \% \mathrm{SDS}$, at $50^{\circ} \mathrm{C}$. The blots were then exposed to film at $-70^{\circ} \mathrm{C}$. Hybridization of Northern blots with a $\beta$-actin cDNA probe indicated equal amounts of poly $(\mathrm{A})^{+}$RNA $(2 \mu \mathrm{g})$ were loaded per lane.

\section{Interspecific Mouse Backcross Mapping}

To establish the chromosomal location of the Eya2 locus, we performed a segregation analysis in the Jackson Laboratory Backcross DNA BSS Panel (C57BL/6JEi $\times$ SPRET/Ei) $\times$ SPRET/ $\mathrm{Ei}$, which has been typed previously for a large number of loci by restriction fragment length polymorphism (RFLP), motifprimed PCR polymorphisms, and microsatellites (Rowe et al. 1994). The map location was determined by the analysis of a polymorphism detected by restriction enzyme digestion of the 306-bp PCR product amplified with the forward and reverse primers (above), which are within the highly conserved region of the gene. This amplicon was subsequently digested by HaeIII and subjected to single-strand conformation polymorphism (SSCP) analysis. PCR conditions were as described (Bucan et al. 1995), except that an 8\% nondenaturing acrylamide gel was used. Detailed mapping data are available at BC Panel Mapping Resource page (http://www.jax.org/resources/ documents/cmdata). Genes linked to Eya2 are Pltp (phospholipid transfer protein; Le Boeuf et al. 1996), Iapt15 (Lueders and Frankel 1994), and Gnas (guanine nucleotide binding protein; Piltz et al. 1992; Wilkie et al. 1993).

Eya2 and Eya3 were then mapped relative to an additional set of described loci (Copeland and Jenkins 1991). Interspecific backcross progeny were generated by mating $(\mathrm{C} 57 \mathrm{BL} / 6 \mathrm{~J} \times$ Mus. spretus $) \mathrm{F}_{1}$ females and C57BL/6J males as described (Copeland and Jenkins 1991). A total of $205 \mathrm{~N}_{2}$ mice were used to map the Eya2 and Eya 3 loci. DNA isolation, restriction enzyme digestion, agarose gel electrophoresis, Southern blot transfer, and hybridization were performed essentially as described (Jenkins et al. 1982). All blots were prepared with Hybond-N+ nylon membrane (Amersham). The probes, a $0.5-\mathrm{kb} E c o \mathrm{RV} / \mathrm{Xhol}$ fragment containing the $3^{\prime}$ untranslated sequences of the mouse M27 clone encoding the Eya2 gene, and a 1.3-kb PstI/Xhol fragment containing the 3 '-untranslated sequences from the mouse M25 clone encoding the Eya3 gene, were labeled with $\left[\alpha^{-}{ }^{32} \mathrm{P}\right] \mathrm{dCTP}$ using a random priming kit (Stratagene); washing was done to a final stringency of $1.0 \times \mathrm{SSCP}, 0.1 \% \mathrm{SDS}, 65^{\circ} \mathrm{C}$. The Eya2 probe detected an EcoRV fragment larger than $23 \mathrm{~kb}$ in C57BL/6J DNA and a 15-kb fragment in EcoRV-digested M. spretus DNA. The presence or absence of the 15-kb EcoRV M. spretus-specific fragment was followed in backcross mice. The Eya3 probe detected 5.5- and 4.3-kb major fragments in PvuII-digested C57BL/6J DNA and 2.4- and 0.9-kb major fragments in PvuIIdigested M. spretus DNA. The 2.4- and 0.9-kb M. spretus fragments cosegregated and their presence or absence was followed in backcross mice.

A description of probes and RFLPs for loci linked to Eya2 and Eya3, including Emv15, $A d a$, the Cebpb, the gap junction protein Gja4, Gardner-Rasheed feline sarcoma viral oncogene homolog $(F g r)$, and the leukemia-associated phosphoprotein ( $\mathrm{Lag}$ ) have been reported previously (Haefliger et al. 1992; Storm et al. 1994; Chen et al. 1995; Jenkins et al. 1995). Recombination distances and gene orders were determined using MapManager (Manley 1993).

\section{Mapping to the Human Chromosomes by FISH}

Screening of a human P1 library (DuPont Merck Pharmaceu- 


\section{ZIMMERMAN ET AL.}

tical Company Human Foreskin Fibroblast P1 Library 1) was performed with PCR primers to the eya gene. DNA from the P1 clone was prepared and labeled with biotin-16-dUTP and hybridized to metaphase spreads of a normal individual as described (Lawrence et al. 1990).

\section{ACKNOWLEDGMENTS}

We thank Anthony Cashmore, Les Klimczak, Laura Lillien, and Neil Shubin for comments and discussions about the manuscript. We thank Debra J. Gilbert for excellent technical assistance, Colin C. Collins (Ph.D.) for screening of the human P1 library, and are grateful to Jonathan Raper, Susannah Chang, Pat Nolan, and Richard Schultz for generously sharing reagents. This research was supported, in part, by Clinical Research grant 6-FY96-0682 from the March of Dimes Birth Defects Foundation (to N.B.S.), a grant from the Fight for Sight Research Division of Prevent Blindness America (to N.B.S.), a Fellowship in IV Drug Abuse Research Center T32DA07241 (to D.L.N.), National Institutes of Health grant HD 28410 (to M.B.), the National Cancer Institute (Department of Health and Human Services) (to N.G.C. and N.A.J.), and a March of Dimes Basil O'Connor Award, the National Eye Institute, and the John Merck Scholars Fund (to N.M.B.).

The publication costs of this article were defrayed in part by payment of page charges. This article must therefore be hereby marked "advertisement" in accordance with 18 USC section 1734 solely to indicate this fact.

\section{REFERENCES}

Ashley, C.T., Jr., and S. Warren. 1995. Trinucleotide repeat expansion and human disease. Annu. Rev. Genet. 29: $703-728$.

Banfi, S., G. Borsani, E. Rossi, L. Bernard, A. Guffanti, F. Rubboli, A. Marchitiello, S. Giglio, E. Coluccia, M. Zollo, O. Zuffardi, and A. Ballabio. 1996. Identification and mapping of human cDNAs homologous to Drosophila mutant genes through EST database searching. Nature Genet. 13: 167-174.

Bonini, N.M. and K.-W. Choi. 1995. Early decisions in Drosophila eye morphogenesis. Curr. Opin. Genet. Dev. 5: 507-515.

Bonini, N.M., W.M. Leiserson, and S. Benzer. 1993. The eyes absent gene: Genetic control of cell survival and differentiation in the developing Drosophila eye. Cell 72: 379-395.

Bucan, M., D. Nagle, R. Hough, V. Chapman, and C. Lo. 1995. Lethality of $R w / R w$ mouse embryos during early postimplantation development. Dev. Biol. 168: 307-318.

Chelsky, D., R. Ralph, and G. Jonak. 1989. Sequence requirements for synthetic peptide-mediated translocation to the nucleus. Mol. Cell. Biol. 9: 2487-2492.

Chen, H., I. Thalmann, J.C. Adams, K.B. Avraham, N.G. Copeland, N.A. Jenkins, D.R. Beier, D.P. Corey, R. Thalmann, and G.M. Duyk. 1995. cDNA cloning, tissue distribution and chromosomal localization of Ecp2, a gene encoding a putative transcription-associated factor predominantly expressed in the auditory organs. Genomics 27: 389-398.

Cheyette, B.N.R., P.J. Green, K. Martin, H. Garren, V. Hartenstein, and S.L. Zipursky. 1994. The Drosophila sine oculis locus encodes a homeodomain-containing protein required for the development of the entire visual system. Neuron 12: 977-996.

Chisholm, A. and H. Horvitz. 1995. Patterning of the Caenorhabditis elegans head region by the Pax-6 family member vab-3. Nature 377: 52-55.

Copeland, N.G. and N.A. Jenkins. 1991. Development and application of a molecular genetic linkage map of the mouse genome. Trends Genet. 7: 113-118.

Dickinson, W. and J. Seger. 1996. Eye evolution. (letter/comment) Science 272: 467-469.

Dingwell, C. and R. Laskey. 1991. Nuclear targeting sequences-A consensus? Trends Biochem. Sci. 16: 478-481.

Gehring, W. 1996. Eye evolution. (letter/comment) Science 272: $468-469$.

Glaser, T., D.S. Walton, and R.L. Maas. 1992. Genomic structure, evolutionary conservation and aniridia mutations in the human PAX6 gene. Nature Genet. 2: 232-239.

Haefliger, J.-A., R. Bruzzone, N.A. Jenkins, D.J. Gilbert, N.G. Copeland, and D.L. Paul. 1992. Four novel members of the connexin family of gap junction proteins: molecular cloning, expression and chromosome mapping. J. Biol. Chem. 267: 2057-2064.

Halder, G., P. Callaerts, and W. Gehring. 1995. Induction of ectopic eyes by targeted expression of the eyeless gene of Drosophila. Science 267: 1788-1792.

Hanson, I. and V. van Heyningen. 1995. Pax6: more than meets the eye. Trends Genet. 11: 268-272.

Haygood, M. 1993. Spreadsheet macros for coloring sequence alignments. BioTechniques 15: 1084-1089.

Heberlein, U. and K. Moses. 1995. Mechanisms of Drosophila retinal morphogenesis: The virtues of being progressive. Cell 81: $987-990$.

Hill, R., J. Favor, B.M. Hogan, C. Ton, G. Saunders, I. Hanson, J. Prosser, T. Jordan, N. Hastie, and V. van Heyningen. 1991. Mouse Small eye results from mutations in a paired-like homeobox-containing gene. Nature 354: 522-525.

Hogan, B., G. Horsburgh, J. Cohen, C. Hetherington, G. Fisher, and M. Lyon. 1986. Small eyes (Sey): A homozygous lethal mutation on chromosome 2 which affects the differentiation of both lens and nasal placodes in the mouse. J. Embryol. Exp. Morphol. 97: 95-110.

Jenkins, N.A., N.G. Copeland, B.A. Taylor, and B.K. Lee. 1982. Organization, distribution and stability of endogenous ecotropic murine leukemia virus DNA 


\section{VERTEBRATE HOMOLOGS OF DROSOPHILA EYES ABSENT}

sequences in chromosomes of Mus musculus. J. Virol. 43: $26-36$.

Jenkins, N.A., D.J. Gilbert, B.C. Cho, M.C. Strobel, S.C. Williams, N.G. Copeland, and P.F. Johnson. 1995. Mouse chromosomal location of the CCAAT/Enhancer binding proteins C/EBP $\beta$ (Cebpb), C/EBPS (Debpd), and CRP1 (Cebpe). Genomics 28: 333-336.

Jordan, T., I. Hanson, D. Zaletayev, S. Hodgson, J. Prosser, A. Seawright, N. Hastie, and V. van Heyningen. 1992. The human PAX6 gene is mutated in two patients with aniridia. Nature Genet. 1: 328-332.

Karlin, S. and C. Burge. 1996. Trinucleotide repeats and long homopeptides in genes and proteins associated with nervous system disease and development. Proc. Natl. Acad. Sci. 93: 1560-1565.

Kozak, M. 1981. Possible role of flanking nucleotides in recognition of the AUG initiator codon by eukaryotic ribosomes. Nucleic Acids Res. 9: 5233-5252.

1984. Compliation and analysis of sequences upstream from the translational start site in eukaryotic mRNAs. Nucleic Acids Res. 12: 857-872.

Lawrence, J., R. Singer, and J. McNeil. 1990. Interphase and metaphase resolution of different distances within the human dystrophin gene. Science 249: 928-932.

LeBoeuf, R., M. Cladwell, A.-Y. Tu, and J. Albers. 1996. Phospholipid transfer protein maps to distal mouse chromosome-2. Genomics 34: 259-260.

Leiserson, W.M., N.M. Bonini, and S. Benzer. 1994. Transvection at the eyes absent gene of Drosophila. Genetics 138: $1171-1179$.

Lueders, K. and W. Frankel. 1994. Mapping of mouse intracisternal A-particle proviral markers in an interspecific backcross. Mamm. Genome 5: 473-478.

Manley, K.F. 1993. A Macintosh program for storage and analysis of experimental genetic mapping data. Genome 4: $303-313$.

Nüsslein-Volhard, C., E. Wiechaus, and H. Kluding. 1984. Mutations affecting the pattern of the larval cuticle in Drosophila melanogaster. I. Zygotic loci on the second chromosome. Wilhelm Roux Arch. Dev. Biol. 193: 267-282.

Oliver, G., A. Mailhos, R. Wehr, N. Copeland, N. Jenkins, and P. Gruss. 1995. Six 3, a murine homologue of the sine oculis gene, demarcates the most anterior border of the developing neural plate and is expressed during eye development. Development 121: 4045-4055.

Pilz, A., P. LeTissier, H. Moseley, J. Peters, and C. Abbott. 1992. Mapping of the structural gene for S-adenosyl homocysteine hydrolase to mouse chromosome 2 , and related sequences to chromosomes 8 and X. Mamm. Genome 3: $633-636$.

Quiring, R., U. Walldorf, U. Kloter, and W.J. Gehring. 1994. Homology of the eyeless gene of Drosophila to the Small eye gene in mice and Aniridia in humans. Science 265: 785-789.
Rowe, L., J. Nadeau, R. Turner, W. Frankel, V. Letts, J. Eppig, M. Ko, S. Thursto, and E. Birkenmeier. 1994. Maps from two interspecific backcross DNA panels available as a community genetic mapping resource. Mamm. Genome 5: $253-274$.

Sambrook, J., E.F. Fritsch, and T. Maniatis. 1989. Molecular cloning: A laboratory manual, 2nd ed. Cold Spring Harbor Laboratory Press, Cold Spring Harbor, NY.

Serikaku, M.A. and J.E. O'Tousa. 1994. sine oculis is a homeobox gene required for Drosophila visual system development. Genetics 138: 1137-1150.

Spence, S.E., D.J. Gilbert, B.S. Harris, M.T. Davisson, N.G. Copeland, and N.A. Jenkins. 1992. Genetic localization of $H a O-1$, blind-sterile $(b s)$, and $E m v-13$ on mouse chromosome 2. Genomics 12: 403-404.

Storm, E., T.V. Huynh, N.G. Copeland, N.A. Jenkins, D.M. Kingsley, and S.-J. Lee. 1994. Limb alterations in brachypodism mice are due to mutations in a new BMP-related member of the TGF- $\beta$ superfamily. Nature 368: 639-643.

Tomlinson, A. 1989. Short-range positional signals in the developing Drosophila eye. Development (Suppl.) 17: 59-63.

Ton, C.C.T., H. Hirvonen, H. Miwa, M.M. Weil, P. Monaghan, T. Jordan, V. van Heyningen, N.D. Hastie, H. Meijers-Heijboer, M. Dreschler, B. Royer-Pokora, F. Collins, A. Swaroop, C.L. Strong, and G.F. Saunders. 1991. Positional cloning and characterization of a paired box- and homeobox-containing gene from the aniridia region. Cell 67: 1059-1074.

Varnum, D. 1983. Blind-sterile: A new mutation on chromosome 2 of the house mouse. J. Hered. 74: 206-207.

Wilkie, T., Y. Chen, D. Gilbert, K. Moore, L. Yu, M. Simon, N. Copeland, and N. Jenkins. 1993. Identification, chromosomal location, and genome organization of mammalian G-protein-coupled receptors. Genomics 18: $175-184$.

Wolff, T. and D.F. Ready. 1993. Pattern formation in the Drosophila retina. In The development of Drosophila melanogaster (ed. M. Bate and A. Martinez-Arias), pp. 1277-1325. Cold Spring Harbor Laboratory Press, Cold Spring Harbor, NY.

Xu, P.-X., I. Woo , H. Her, D. Beier, and R.L. Maas. 1997. Mouse Eya homologues of the Drosophila eyes absent gene require Pax6 for expression in cranial placodes. Development 124: $219-231$.

Zhang, Y. and S. Emmons. 1995. Specification of sense-organ identity by a Caenorhabditis elegans Pax- 6 homologue. Nature 377: 55-59.

Zipursky, S.L. and G.M. Rubin. 1994. Determination of neuronal cell fate: Lessons from the R7 neuron of Drosophila. Annu. Rev. Neurosci. 17: 373-397.

Received November 11, 1996; accepted in revised form December 23, 1996. 


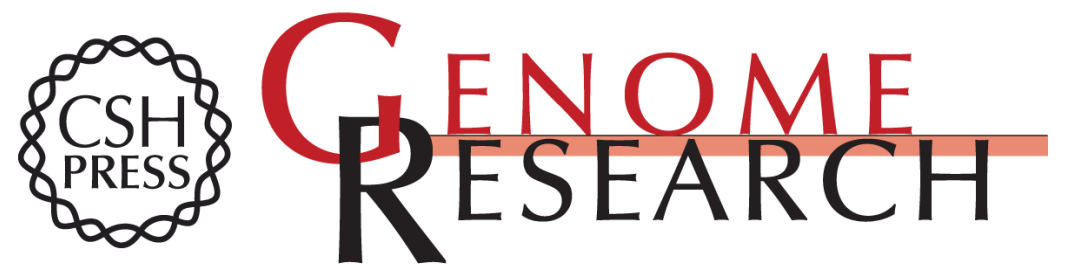

\section{Cloning and characterization of two vertebrate homologs of the Drosophila eyes absent gene.}

J E Zimmerman, Q T Bui, E Steingrímsson, et al.

Genome Res. 1997 7: 128-141

Access the most recent version at doi:10.1101/gr.7.2.128

References This article cites 47 articles, 12 of which can be accessed free at:

http://genome.cshlp.org/content/7/2/128.full.html\#ref-list-1

\section{License}

Email Alerting Receive free email alerts when new articles cite this article - sign up in the box at the Service top right corner of the article or click here.

\section{Affordable, Accurate Sequencing.}

To subscribe to Genome Research go to:

https://genome.cshlp.org/subscriptions 